\title{
Magnetic properties and spin kinetics of a heavy-fermion Kondo lattice
}

\author{
B.I. Kochelaev \\ Institute of Physics, Kazan Federal University, Kremlevskaya 18, Kazan 42008, Russian Federation \\ E-mail: bkochelaev@gmail.com
}

Received July 8, 2016, published online November 25, 2016

\begin{abstract}
A review of peculiarities of magnetic properties and spin kinetics of a heavy-fermion Kondo lattice revealed by electron spin resonance (ESR) experiments and their theoretical analysis is given. Among the issues discussed in some detail are the renormalization of spin kinetics coefficients due to the Kondo effect, formation of the collective spin modes of the Kondo ions and wide-band conduction electrons, unexpected behavior of ESR parameters as functions of temperature and magnetic fields. Special attention is focused on the possible role of the Kondo effect for the ESR signal existence at low temperatures.
\end{abstract}

PACS: $76.30 . \mathrm{Kg}$ Rare-earth ions and impurities;

71.27.+a Strongly correlated electron systems; heavy fermions;

75.40.Gb Dynamic properties (dynamic susceptibility, dynamic scaling).

Keywords: magnetic resonance, heavy fermions, Kondo effect.

\section{Introduction}

Heavy fermion compounds like $\mathrm{YbRh}_{2} \mathrm{Si}_{2}$ and $\mathrm{YbIr}_{2} \mathrm{Si}_{2}$ have very peculiar magnetic, thermal, transport and spin kinetics properties. They are determined by the interplay of the strong repulsion of $4 f$ electrons on the rareearth ion sites, their hybridization with wide-band conduction electrons and the influence of the crystalline electrical field. Experimental observations are consistent with a metallic behavior with very heavy charge carriers having the properties of a Landau Fermi liquid (LFL). With decreasing of temperature these materials experience the Kondo effect. At very low temperatures a long-range antiferromagnetic (AF) order appears with $T_{N}=70 \mathrm{mK}$ in $\mathrm{YbRh}_{2} \mathrm{Si}_{2}$, which is suppressed by an external magnetic field at the quantum critical point (QCP) with $H_{c}=0.06 \mathrm{~T}, T \rightarrow 0$. Near the QCP at higher magnetic fields and up to surprisingly high temperatures, a new phase appears exhibiting non-Fermi-liquid (NFL) behavior. In this temperature region also some features of ferromagnetic fluctuations were observed. The electrical resistivity in $\mathrm{YbRh}_{2} \mathrm{Si}_{2}$ linearly increases with temperature and the Sommerfeld coefficient of the electronic specific heat diverges logarithmically upon cooling down to $0.3 \mathrm{~K}$. The phase diagram based on these experimental findings is presented in Fig. 1 [1].

The discovery of electron spin resonance (ESR) in the $\mathrm{YbRh}_{2} \mathrm{Si}_{2}$ compound at temperatures below the thermodynamically determined Kondo temperature $\left(T_{K}=25 \mathrm{~K}\right)$ became a great surprise for the condensed matter physics community [2]. According to the common belief, based on the single ion Kondo effect, the ESR signal should not be

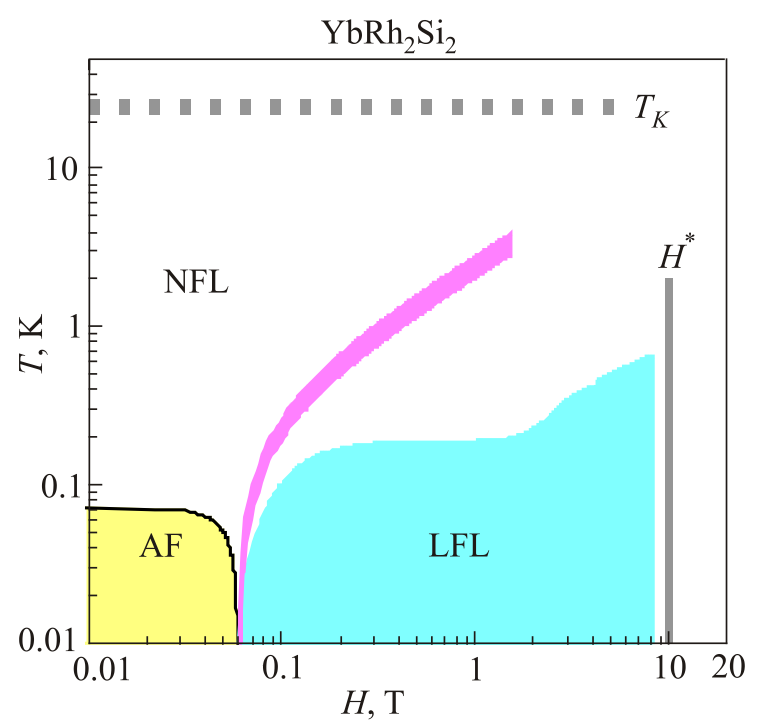

Fig. 1. (Color online) Experimental phase diagram of $\mathrm{YbRh}_{2} \mathrm{Si}_{2}$ for $\mathbf{H} \perp c$ from [1]. $T_{K}$ and $H^{*}$ mark the crossover from local moment to heavy fermion behavior, derived from the zero-field specific heat and low-temperature magnetization. LFL and NFL denote the Landau Fermi-liquid and non-Fermi-liquid regions, respectively. The broad pink line specifies the position of crossover in the isothermal Hall resistivity, isothermal magnetostriction, magnetization and longitudinal resistivity. AF means the antiferromagnetic phase. 
observable by at least two reasons. Firstly, the magnetic moments of the Kondo ions should be screened by the conduction electrons at temperatures below $T_{K}$; secondly, the ESR linewidth was expected to be of the order of $T_{K}$, i.e. $500 \mathrm{GHz}$. The experimental results were completely opposite: at $X$-band $(9.4 \mathrm{GHz})$ and $T=1.6 \mathrm{~K}$ a linewidth of $0.3 \mathrm{GHz}$ was observed (see Fig. 2 from [3], see also [4]) and the ESR intensity corresponds to the participation of all Kondo ions with a temperature dependence following a Curie-Weiss law [5]. In addition, the angular dependence of the resonant magnetic field reflects the tetragonal symmetry of the crystal electric field at the position of the $\mathrm{Yb}$ ion with extremely anisotropic $g$ factors $g_{\perp}=3.56$, $g_{\|}=0.17$ at $T=5 \mathrm{~K}$ [2]. Similar results were obtained later for $\mathrm{YbIr}_{2} \mathrm{Si}_{2}[6,7]$, and the main features of the ESR phenomenon were confirmed for $\mathrm{YbRh}_{2} \mathrm{Si}_{2}$ at very high frequencies up to $360 \mathrm{GHz}$ [8]. This discovery stimulated considerable efforts, both theoretical and experimental, in order to understand the nature of the ESR existence and Kondo lattice systems in general.

The first attempt was made by Zvyagin et al. using a model of metallic systems with multichannel Kondo magnetic impurities [9] and the Anderson lattice model [10] correspondingly, relating them to the actual compound on a qualitative level. Abrahams and Wölfle [11,12] studied the ESR in heavy-fermion systems using a Fermi-liquid description in the framework of the Anderson model, where the local magnetic moment is that of a quasilocalized $f$ electron. Schlottmann [13] suggested an explanation of the ESR signal existence based on the Kondo lattice model with an isotropic interaction between the

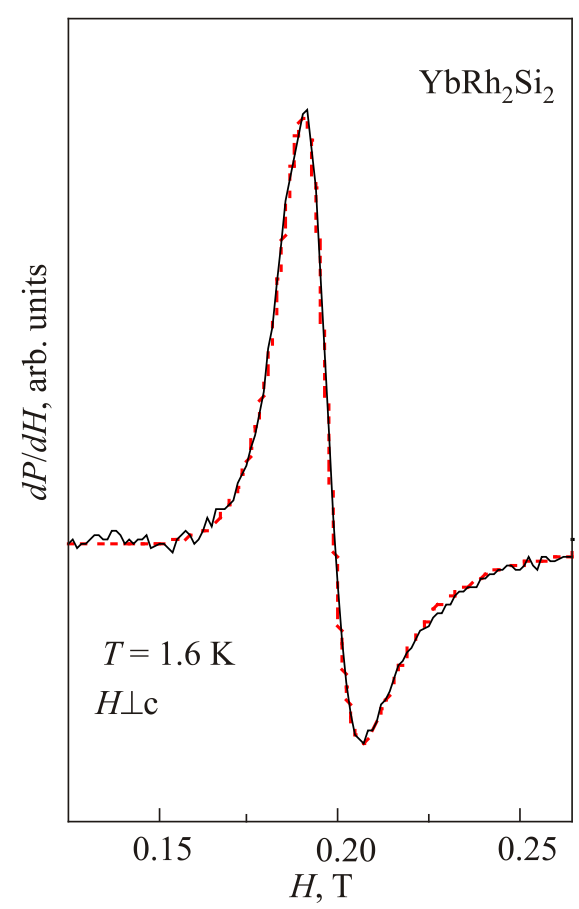

Fig. 2. Representative ESR spectrum in $\mathrm{YbRh}_{2} \mathrm{Si}_{2}$ at $T=1.6 \mathrm{~K}$ (from [3]). conduction electrons and the Kondo ions. However, both of these approaches do not take into account the strong spin orbital interaction and the crystal electric field (CEF) effects which result in the anisotropy of the Kondo interaction similar to that of the Zeeman energy. On the contrary a semi-phenomenological theory presented by Huber [14] takes into account the anisotropy of the static and dynamical susceptibilities. The author was able to describe the ESR data in $\mathrm{Yb}$ heavy-fermion compounds, especially their angular dependence, but the analysis did not touch the problem of the ESR signal existence, assuming it a priori.

This paper presents a short review of magnetic properties and spin kinetics of a heavy-fermion Kondo lattice including a problem of the ESR existence. This analysis is based on a model of localized $4 f$ electrons coupled to the wide-band conduction electrons as a starting point. It was found that the key ingredient of the ESR signal existence in a heavy-fermion Kondo lattice in the NFL state is a formation of a collective spin mode of quasi-localized $f$ electrons and wideband conduction electrons even in a strongly anisotropic system. In opposite to a common belief, it was found that the Kondo effect can support the ESR existence.

\section{Crystal electric field and magnetic susceptibility}

The angle resolved photoemission spectroscopy (ARPES) measurements on $\mathrm{YbRh}_{2} \mathrm{Si}_{2}$ revealed a very narrow $4 f$ band near the Fermi energy [15]. It is an additional argument confirming the quasi-localized nature of the $f$-electron motion (in the case of absolutely localized electrons their width of energy band as a function of the wave vector reduces to zero). So, it was reasonable to investigate the energy spectra, $g$ factors of the ground state and the static magnetic susceptibility of the $\mathrm{Yb}^{3+}$ ions in the $\mathrm{YbRh}_{2} \mathrm{Si}_{2}$ and $\mathrm{YbIr}_{2} \mathrm{Si}_{2}$ compounds with localized $f$ electrons. A full solution of this problem was given in the papers [16-18]. Here we describe a scheme of calculations and the main results.

A free $\mathrm{Yb}^{3+}$ ion has the electronic $4 f^{13}$ configuration with one term ${ }^{2} F$. The spin-orbit interaction splits the ${ }^{2} F$ term into two multiplets: ${ }^{2} F_{7 / 2}$ with $J=7 / 2$ and ${ }^{2} F_{5 / 2}$ with $J=5 / 2$, where $J$ denotes the total momentum $\mathbf{J}=\mathbf{L}+\mathbf{S}$ with $\mathbf{L}$ and $\mathbf{S}$ as the orbital and spin momentum of the ion. The excited multiplet ${ }^{2} F_{5 / 2}$ is separated from the ground one ${ }^{2} F_{7 / 2}$ by about $1 \mathrm{eV}$. Since this value is much larger than the energy of the crystal electric field, we consider the ground multiplet only. The potential of the tetragonal crystal field for an ion can be written as

$$
V=\alpha B_{2}^{0} O_{2}^{0}+\beta\left(B_{4}^{0} O_{4}^{0}+B_{4}^{4} O_{4}^{4}\right)+\gamma\left(B_{6}^{0} O_{6}^{0}+B_{6}^{4} O_{6}^{4}\right) .
$$

The crystal field parameters are $B_{k}^{q}$. The operators $O_{k}^{q}$ and coefficients $\alpha, \beta, \gamma$ are standard and given in the book by Abragam and Bleaney [19]. To define energy levels and wave functions of the $\mathrm{Yb}^{3+}$ ion one has to diagonalize the matrix of the operator (1) on the states of the ground 
multiplet ${ }^{2} F_{7 / 2}$. As a result, the crystal field splits the lower multiplet into four Kramers doublets. Inelastic neutron scattering (INS) experiments give the following values for the excited energy levels in $\mathrm{YbRh}_{2} \mathrm{Si}_{2}: \Delta_{1}=17 \mathrm{meV}$, $\Delta_{2}=25 \mathrm{meV}, \Delta_{3}=43 \mathrm{meV}$ relative to the ground doublet [20]. These results together with mentioned ARPES and ESR data gave an opportunity to find the single set of tetragonal CEF parameters for $\mathrm{Yb}^{3+}$ ion in $\mathrm{YbRh}_{2} \mathrm{Si}_{2}$, presented in the Table 1 [18].

Table 1. Crystal electric field parameters $B_{k}^{q}(\mathrm{meV})$ for $\mathrm{Yb}^{3+}$ ion in $\mathrm{YbRh}_{2} \mathrm{Si}_{2}$ [18]

\begin{tabular}{c|c|c|c|c}
\hline \hline$B_{2}^{0}$ & $B_{4}^{0}$ & $B_{4}^{4}$ & $B_{6}^{0}$ & $B_{6}^{4}$ \\
\hline 22.4 & -6.6 & \pm 29.6 & -2.3 & \pm 63.9 \\
\hline \hline
\end{tabular}

The Zeeman energy of the $\mathrm{Yb}$ ion $H_{\mathrm{ZJ}}$ for the ground multiplet ${ }^{2} F_{7 / 2}$ can be expressed via the total momentum of the ion. Using the Lande $g$ factor $g_{J}=8 / 7$, we have

$$
H_{\mathrm{Z} J}=\sum_{i} \mu_{B}\left(\mathbf{L}_{i}+2 \mathbf{S}_{i}\right) \mathbf{H}=\sum_{i} g_{J} \mu_{B} \mathbf{J}_{i} \mathbf{H},
$$

where $\mathbf{H}$ is the external magnetic field and $\mu_{B}$ is the Bohr magneton. The calculations of the static magnetic susceptibility can be performed in a straightforward way. In the case of low enough temperatures $k_{B} T \ll \Delta_{k}$ we have the standard expressions for two following contributions to the magnetic susceptibility $\chi=\chi_{\mathrm{C}}+\chi_{\mathrm{VV}}$ :

$$
\begin{aligned}
& \chi_{\alpha \beta}(\mathrm{C})=\frac{N\left(g_{J} \mu_{B}\right)^{2}}{2 k_{B} T} \sum_{v v^{\prime}}\left\langle l v\left|J_{\alpha}\right| l v^{\prime}\right\rangle\left\langle l v^{\prime}\left|J_{\beta}\right| l v\right\rangle \equiv \frac{C_{\alpha \beta}^{0}}{T} ; \\
& \chi_{\alpha \beta}(\mathrm{VV})=N\left(g_{J} \mu_{B}\right)^{2} \sum_{\substack{v v^{\prime} \\
n(\neq l)}} \frac{\left\langle l v\left|J_{\alpha}\right| n v^{\prime}\right\rangle\left\langle n v^{\prime}\left|J_{\beta}\right| l v\right\rangle}{E_{n}-E_{l}} .
\end{aligned}
$$

Here $N$ is the density of the $\mathrm{Yb}$ ions in the crystal. Indexes $n, v, v^{\prime}$ denote the Kramers doublets and their corresponding components; $|l v\rangle$ is the ground Kramers doublet. The first term, $\chi_{\alpha \beta}(C)$, corresponds to the Curie susceptibility proportional to the inverse temperature, while the second term, $\chi_{\alpha \beta}(\mathrm{VV})$, corresponds to the Van Vleck susceptibility, which does not depend on temperature. According to the tetragonal symmetry from all the Curie constants survive two ones only: $C_{\|}^{0}$ and $C_{\perp}^{0}$.

Before a comparison of these results with an experiment, one should take into account their renormalization due to interaction of the $\mathrm{Yb}$ ions with conduction electrons and their indirect exchange interaction via these electrons, socalled RKKY interaction. One could expect, that the temperature dependence should become a Curie-Weiss type:

$$
\chi_{\|, \perp}=\frac{C_{\|, \perp}}{T+\theta_{\|, \perp}}+\chi_{\|, \perp}(\mathrm{VV})
$$

Here $\theta_{\|, \perp}$ are the Weiss constants.
It is appropriate to mention at this point, that there is a different approach to calculations of the static magnetic susceptibility in these materials, so-called a locally quantum critical scenario [21,22]. One of the hallmarks of this local criticality is a generalized Curie-Weiss law, which for a wave-vector-dependent magnetic susceptibility can be written in the form

$$
\chi(\mathbf{q}, T)=\frac{C}{T^{\alpha}+\theta_{\mathbf{q}}^{\alpha}}
$$

with an exponent $\alpha<1$. In particular, experimental results for the $\mathrm{YbRh}_{2} \mathrm{Si}_{2}$ magnetic susceptibility in the temperature region $0.3 \mathrm{~K}<T<10 \mathrm{~K}$ was related to this scenario for $\mathbf{q}=0$, with $\alpha=0.6, \theta=0$ [22].

Figure 3 shows the experimental results for the static magnetic susceptibility in $\mathrm{YbRh}_{2} \mathrm{Si}_{2}$ in the temperature region $0.02 \mathrm{~K}<T<3.6 \mathrm{~K}$ from [22] together with a fitting according formulas (4) and (5). The fitted constants in (4) were $C_{\perp}=2.31 \cdot 10^{-6} \mathrm{~m}^{3} \cdot \mathrm{mol}^{-1} \cdot \mathrm{K}$ and $\theta_{\perp}=0.22 \mathrm{~K}$ (solid line). One can see that the temperature dependence of magnetic susceptibility is better described in the frame of a simple and transparent local model of the $\mathrm{Yb}^{3+}$ ions in the crystal electric field in comparison with a sophisticated scenario of the local quantum criticality. However, in spite of the success of this entirely local approach for the static magnetic susceptibility of $\mathrm{YbRh}_{2} \mathrm{Si}_{2}$, such a model is insufficient for a proper theoretical understanding of the dynamical susceptibilities as observed by ESR. In the following sections this problem will be considered by taking into account an interaction of the $\mathrm{Yb}^{3+}$ ions with wide-band conduction electrons and their collective response to the resonant magnetic alternating field (the bottleneck regime). This interaction will be considered in details, including a role of the Kondo effect in a formation of the collective spin modes.

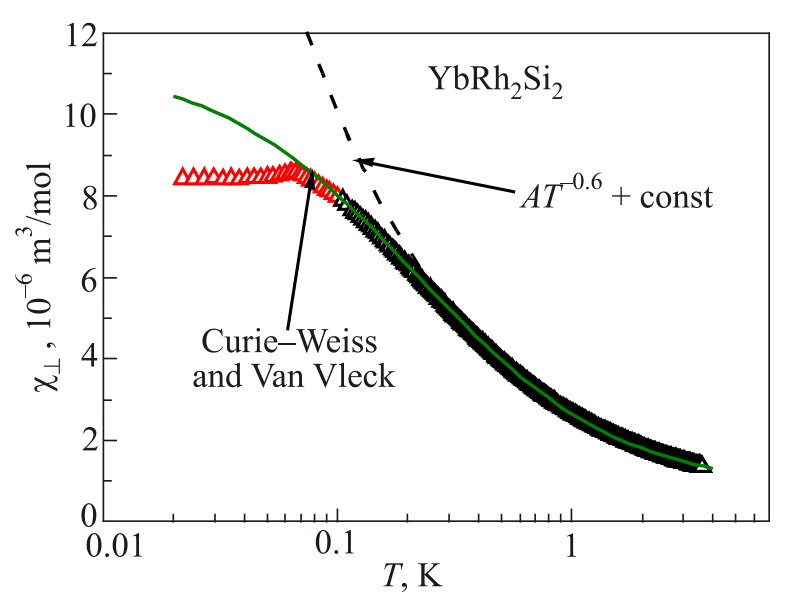

Fig. 3. (Color online) Fitting of the magnetic ac-susceptibility data [22] by expression (4) (solid line) and by (5) (dashed line) with parameters given in the text (from [16]). 


\section{Dynamical magnetic susceptibility}

\subsection{Theoretical model}

To consider the spin dynamics in the Kondo lattice under discussion we use the model including the kinetic energy of conduction electrons, the Zeeman energy, the Kondo interaction between $\mathrm{Yb}$ ions and conduction electrons, and the coupling between the $\mathrm{Yb}$ ions via conduction electrons (RKKY interaction) [23-30]. The physics of lowenergy spin excitations at temperatures $T \ll 200 \mathrm{~K}$ can be described by the lowest Kramers doublet. It means that the Zeeman energy (2) has to be projected onto this state. Within the ground Kramers doublet, described by the wave functions of the type $|l \uparrow, \downarrow\rangle$, the Zeeman energy can be represented by the effective spin Hamiltonian with an effective spin $S=1 / 2$ :

$$
\begin{gathered}
H_{Z S}=\sum_{i}\left[g_{\perp}\left(B_{x} S_{i}^{x}+B_{y} S_{i}^{y}\right)+g_{\|} B_{z} S_{i}^{z}\right] ; \\
g_{\perp}=g_{J}\left\langle l \uparrow\left|J_{x}+i J_{y}\right| l \downarrow\right\rangle, \quad g_{\|}=2 g_{J}\left\langle l \uparrow\left|J_{z}\right| l \downarrow\right\rangle .
\end{gathered}
$$

Here the $z$ axis is along the crystal symmetry and we have introduced $B=\mu_{B} H$. We can see that starting from the Zeeman energy with the isotropic Lande $g$ factor, we came to the anisotropic expression.

The Kondo exchange coupling of the rare-earth ion with the conduction electrons occurs due to the hybridization of their wave functions at the ion site. An exchange integral can be written in the form

$$
\begin{aligned}
& A\left(\mathbf{k}, \mathbf{k}^{\prime}\right)=\sum_{i} \int \psi^{*}\left(\mathbf{r}^{\prime}, \mathbf{k}\right) \Psi_{4 f}\left(\mathbf{r}_{1}, \ldots, \mathbf{r}_{i}, \ldots, \mathbf{r}_{n}\right) \frac{e^{2}}{\left|\mathbf{r}_{i}-\mathbf{r}^{\prime}\right|} \times \\
& \times \psi\left(\mathbf{r}_{i}, \mathbf{k}^{\prime}\right) \Psi_{4 f}\left(\mathbf{r}_{1}, \ldots, \mathbf{r}^{\prime}, \ldots, \mathbf{r}_{n}\right) d \mathbf{r}^{\prime} d \mathbf{r}_{1} \ldots d \mathbf{r}_{n} .
\end{aligned}
$$

Here $\psi(\mathbf{r}, \mathbf{k})$ is the Bloch wave function of the conduction electrons. The wave function of the $4 f$ electrons $\Psi_{4 f}$ is represented by the determinant constructed from the oneelectron wave functions of the type $R_{4 f}(r) Y_{3}^{m}(r)$. Expanding the Bloch functions and $\left|\mathbf{r}_{i}-\mathbf{r}^{\prime}\right|^{-1}$ in spherical harmonics, one can obtain $A\left(\mathbf{k}, \mathbf{k}^{\prime}\right)$ as an expansion in multipoles. The small parameter of this expansion is the value $k_{F}\left\langle r_{4 f}\right\rangle \ll 1$, the product of the wave vector of the conduction electron at the Fermi surface and an average radius of the $4 f$ electron. The Kondo interaction corresponding to the zero order of this expansion is isotropic and can be written in the form

$$
H_{K J}=A_{0} \sum_{i} \mathbf{S}_{i} \boldsymbol{\sigma}_{i}=A_{0}\left(g_{J}-1\right) \sum_{i} \mathbf{J}_{i} \boldsymbol{\sigma}_{i},
$$

where $\boldsymbol{\sigma}_{i}$ is the spin density of the conduction electrons at the ion site. The next terms of the expansion in multipoles are much smaller, and we neglect them. The projection of the isotropic Kondo interaction (8) involves the same matrix elements of the total momentum $\mathbf{J}$ as in the case of the Zeeman energy. Hence, after projection onto the ground Kramers state the total Kondo interaction can be expressed via the $g$ factors, given above

$$
\begin{gathered}
H_{K}=\sum_{i}\left[J_{\perp}\left(S_{i}^{x} \sigma_{i}^{x}+S_{i}^{y} \sigma_{i}^{y}\right)+J_{\|} S_{i}^{z} \sigma_{i}^{z}\right] ; \\
J_{\perp}=A_{0} \frac{g_{J}-1}{g_{J}} g_{\perp}, \quad J_{\|}=A_{0} \frac{g_{J}-1}{g_{J}} g_{\|} .
\end{gathered}
$$

The same arguments can be used to reveal the anisotropy of the RKKY interaction between the Kondo ions. Although this interaction appears in the second order of the Kondo interaction (8), it is convenient to consider it independently. Starting with the isotropic exchange Hamiltonian for two Kondo ions $H_{i j}^{\mathrm{ex}}=I_{i j}^{\mathrm{ex}} \mathbf{J}_{i} \mathbf{J}_{j}$, we arrive after projection to the effective anisotropic exchange interaction

$$
\begin{aligned}
& H_{\mathrm{ex}}=\frac{1}{2} \sum_{i j}\left[I_{i j}^{\perp}\left(S_{i}^{x} S_{j}^{x}+S_{i}^{y} S_{j}^{y}\right)+I_{i j}^{\|} S_{i}^{z} S_{j}^{z}\right] ; \\
& I_{i j}^{\perp}=\left(g_{\perp} / g_{J}\right)^{2} I_{i j}^{\mathrm{ex}}, \quad I_{i j}^{\|}=\left(g_{\|} / g_{J}\right)^{2} I_{i j}^{\mathrm{ex}} .
\end{aligned}
$$

This expression was given earlier in [31]. As a matter of fact all these results are simply consequences of the wellknown Wigner-Eckart theorem.

The kinetic energy of the conduction electrons and their Zeeman energy can be written as

$$
\begin{gathered}
H_{c}=\sum_{\mathbf{k} v} \varepsilon_{\mathbf{k}} c_{\mathbf{k} v}^{+} c_{\mathbf{k} v}, \quad H_{Z \sigma}=\sum_{j} g_{\sigma} \mathbf{B} \boldsymbol{\sigma}_{j} \\
\boldsymbol{\sigma}_{j}=\sum_{\mathbf{k} \mathbf{k}^{\prime} v v^{\prime}} \mathbf{s}_{v v^{\prime}} \mathrm{e}^{i\left(\mathbf{k}^{\prime}-\mathbf{k}\right) \mathbf{r}_{j}} c_{\mathbf{k} v}^{+} c_{\mathbf{k}^{\prime} v^{\prime}}
\end{gathered}
$$

The energy band of conduction electrons is assumed symmetric about the Fermi surface, and their energy relative to the Fermi level $\varepsilon_{\mathbf{k}}$ is running within limits $(-W, W)$. Here $c_{\mathbf{k} v}^{+}, c_{\mathbf{k} v}$ are the creation and annihilation operators of the conduction electrons with the wave vector and spin projection $(\mathbf{k}, v), g_{\sigma}$ denotes their $g$ factor, $\mathbf{s}_{v v^{\prime}}$ are the matrix elements of the electron spin operator for $s=1 / 2$. The Kondo interaction in this representation takes the form

$$
\begin{aligned}
H_{K} & =\frac{1}{2} \sum_{j \mathbf{k} \mathbf{k}^{\prime}}\left[J_{\perp}\left(S_{j}^{+} c_{\mathbf{k} \downarrow}^{+} c_{\mathbf{k}^{\prime} \uparrow}+S_{j}^{-} c_{\mathbf{k} \uparrow}^{+} c_{\mathbf{k}^{\prime} \downarrow}\right)+\right. \\
& \left.+J_{\|} S_{j}^{z}\left(c_{\mathbf{k} \uparrow}^{+} c_{\mathbf{k}^{\prime} \uparrow}-c_{\mathbf{k} \downarrow}^{+} c_{\mathbf{k}^{\prime} \downarrow}\right)\right] \mathrm{e}^{i\left(\mathbf{k}^{\prime}-\mathbf{k}\right) \mathbf{r}_{j}} .
\end{aligned}
$$

Here $S_{j}^{ \pm}=S_{j}^{x} \pm i S_{j}^{y}$.

\subsection{Spin dynamics}

The most detailed ESR experiments with the $\mathrm{YbRh}_{2} \mathrm{Si}_{2}$ samples were performed for the static and alternating magnetic fields oriented perpendicular to the crystal symmetry 
axis, since the $g$ factor along this axis is very small $\left(g_{\|}<0.2\right)$. So, as a first step we consider in detail the spin dynamics of the system for this case with the $z$ axis along the static magnetic field. After a unitary transformation to a new coordinate system, the Hamiltonian takes the form $H=H_{0}+H_{K}+H_{\text {ex }}$ :

$$
\begin{aligned}
& H_{0}=\sum_{\mathbf{k} v} \varepsilon_{\mathbf{k}} c_{\mathbf{k} v}^{+} c_{\mathbf{k} v}+B \sum_{i}\left(g_{\perp} S_{i}^{z}+g_{\sigma} \sigma_{i}^{z}\right), \\
& H_{K}=\sum_{i}\left[J_{\perp}\left(S_{i}^{x} \sigma_{i}^{x}+S_{i}^{z} \sigma_{i}^{z}\right)+J_{\|} S_{i}^{y} \sigma_{i}^{y}\right], \\
& H_{\mathrm{ex}}=\frac{1}{2} \sum_{i j}\left[I_{i j}^{\perp}\left(S_{i}^{x} S_{j}^{x}+S_{i}^{z} S_{j}^{z}\right)+I_{i j}^{\|} S_{i}^{y} S_{j}^{y}\right] .
\end{aligned}
$$

The ESR response is given by the transverse (relative to $z$ axis) dynamical susceptibility for a total magnetization of the $\mathrm{Yb}$ ions and conduction electrons

$$
\chi(\omega)=\sum_{\alpha \beta} \chi_{\alpha \beta}(\omega) ; \quad \alpha, \beta=s, \sigma .
$$

Partial susceptibilities $\chi_{\alpha \beta}(\omega)$ can be represented by the Green functions

$$
\begin{aligned}
& \chi_{s s}=-g_{\perp}^{2}\left\langle\left\langle S^{-} \mid S^{+}\right\rangle\right\rangle, \quad \chi_{s \sigma}=-g_{\perp} g_{\sigma}\left\langle\left\langle S^{-} \mid \sigma^{+}\right\rangle\right\rangle, \\
& \chi_{\sigma s}=-g_{\sigma} g_{\perp}\left\langle\left\langle\sigma^{-} \mid S^{+}\right\rangle\right\rangle, \chi_{\sigma \sigma}=-g_{\sigma}^{2}\left\langle\left\langle\sigma^{-} \mid \sigma^{+}\right\rangle\right\rangle .
\end{aligned}
$$

Here $\langle\langle A \mid B\rangle\rangle$ is the Fourier transform of the retarded commutation Green function

$$
\langle\langle A \mid B\rangle\rangle=-i \int_{0}^{\infty} d t \exp (i \omega t)\langle[A(t), B(0)]\rangle .
$$

$\mathbf{S}, \boldsymbol{\sigma}$ are the total spin operators of $\mathrm{Yb}$ ions and conduction electrons; $S^{ \pm}=\left(S^{x} \pm i S^{y}\right), \sigma^{ \pm}=\left(\sigma^{x} \pm i \sigma^{y}\right)$, respectively, and $\langle\ldots\rangle$ means the statistical average at temperature $T$.

The Green functions can be calculated by the perturbation method. As a result a set of coupled equations appears which is convenient to write in matrix form

$$
\left(\begin{array}{ll}
a_{s s} & a_{s \sigma} \\
a_{\sigma s} & a_{\sigma \sigma}
\end{array}\right)\left(\begin{array}{ll}
\chi_{s s} & \chi_{s \sigma} \\
\chi_{\sigma s} & \chi_{\sigma \sigma}
\end{array}\right)=\left(\begin{array}{cc}
g_{\perp}^{2}\left\langle S^{z}\right\rangle & 0 \\
0 & g_{\sigma}^{2}\left\langle\sigma^{z}\right\rangle
\end{array}\right)
$$

with

$$
\begin{array}{ll}
a_{s s}=\omega-\omega_{s}+\Sigma_{s s}, & a_{s \sigma}=g_{\perp}^{2}\left\langle S^{z}\right\rangle \lambda-\frac{g_{\perp}}{g_{\sigma}} \Sigma_{s \sigma}, \\
a_{\sigma s}=g_{\sigma}^{2}\left\langle\sigma^{z}\right\rangle \lambda-\frac{g_{\sigma}}{g_{\perp}} \Sigma_{\sigma s}, & a_{s s}=\omega-\omega_{\sigma}+\Sigma_{\sigma \sigma} .
\end{array}
$$

Here $\quad \lambda=\left(J_{\perp}+J_{\|}\right) / g_{\perp} g_{\sigma}, \quad\left\langle S^{z}\right\rangle=-g_{\perp} B / 4(T+\theta)$, $\left\langle\sigma^{z}\right\rangle=-g_{\sigma} \rho B / 2, \rho$ is the conduction electron density of states at the Fermi surface. The resonance frequencies of $\mathrm{Yb}$ ions and conduction electrons, containing the first order
Knight shifts due to the Kondo and RKKY interactions are given by

$$
\begin{aligned}
& \omega_{S}=g_{\perp}\left(B+J_{\perp}\left\langle\sigma^{z}\right\rangle\right)+4 \theta\left\langle S^{z}\right\rangle, \\
& \omega_{\sigma}=g_{\sigma} B+J_{\perp}\left\langle S^{z}\right\rangle .
\end{aligned}
$$

Here $\theta$ is the Weiss temperature due to the RKKY interaction in a molecular field approximation

$$
\theta=\frac{1}{4} \sum_{j} I_{i j}^{\|}
$$

The imaginary part of the kinetic coefficient $\Gamma_{s S}=\operatorname{Im}\left(\Sigma_{s S}\right)$ describes the spin relaxation of $\mathrm{Yb}$ ions toward conduction electrons, which remain in a thermodynamically equilibrium state (the Korringa relaxation rate). The Overhauser relaxation rate (conduction electrons relax toward $\mathrm{Yb}$ spin system, being in the equilibrium with the thermal bath) is described by the kinetic coefficient $\Gamma_{\sigma \sigma}=\operatorname{Im}\left(\Sigma_{\sigma \sigma}\right)$. Calculations of these coefficients up to the third order in the Kondo interaction give [24]

$$
\begin{aligned}
& \Gamma_{s s}=\frac{\pi}{4} \rho^{2} T\left[3 J_{\perp}^{2}+J_{\|}^{2}+8 \rho J_{\|} J_{\perp}^{2} \ln (W / T)\right], \\
& \Gamma_{\sigma \sigma}=\frac{\pi}{8} \frac{g_{\sigma} \rho T}{g_{\perp}(T+\theta)}\left[3 J_{\perp}^{2}+J_{\|}^{2}+8 \rho J_{\|} J_{\perp}^{2} \ln (W / T)\right],
\end{aligned}
$$

where $W$ is a half of the band width of the conduction electrons. Besides, for a correct analysis of a stationary solution one has to take into account the spin relaxation of Kondo ions and conduction electrons toward the thermal bath ("lattice"). Correspondingly, the relaxation rates $\Gamma_{s s}$, $\Gamma_{\sigma \sigma}$ in equations (18) should be replaced by $\Gamma_{s s}+\Gamma_{s L}$ and $\Gamma_{\sigma \sigma}+\Gamma_{\sigma L}$, respectively.

\section{Collective spin modes of localized moments and conduction electrons}

An equilibrium state approximation for the conduction electron spin system is not valid to study the ESR response of the samples with a high concentration of Kondo ions. It is especially the case for a Kondo lattice. Instead, one has to treat the coupled kinetic equations (17) for both magnetic moments of Kondo ions and conduction electrons. Two additional kinetic coefficients $\Sigma_{s \sigma}$ and $\Sigma_{\sigma s}$ couple the kinetic equations of motion for the transverse magnetizations of localized moments and conduction electrons. Their imaginary parts represent additional relaxation rates:

$$
\begin{aligned}
& \Gamma_{s \sigma}=\frac{\pi}{4} \frac{g_{\perp} \rho T}{g_{\sigma}(T+\theta)} J_{\perp}\left(J_{\perp}+J_{\|}\right)\left[1+\rho\left(J_{\perp}+J_{\|}\right) \ln (W / T)\right], \\
& \Gamma_{\sigma s}=\frac{\pi}{2} \rho^{2} T J_{\perp}\left(J_{\perp}+J_{\|}\right)\left[1+\rho\left(J_{\perp}+J_{\|}\right) \ln (W / T)\right] .
\end{aligned}
$$

To study the ESR response of the total system we have to find solutions of the system (17). The poles of the total 
dynamical susceptibility are determined by the condition $a_{s s} a_{\sigma \sigma}-a_{s \sigma} a_{\sigma s}=0$. As the result we obtain two complex roots: their real parts represent resonance frequencies, their imaginary parts represent the corresponding relaxation rates. We are interested in a solution close to the Kondo ion resonance frequency $\omega_{s}$.

The coupling between the two systems is especially important if the relaxation of conduction electrons toward the Kondo ions is much faster than to the lattice and the resonant frequencies are close to one another (a relaxation dominated "bottleneck" regime):

$$
\Gamma_{s s} \gg\left(\Gamma_{s L}+\Gamma_{\sigma L}\right),\left|\omega_{s}-\omega_{\sigma}\right|
$$

In the case of an isotropic system and equal Larmor frequencies $g_{\perp}=g_{\|}=g_{\sigma}$ the ESR linewidth in the bottleneck regime is greatly narrowed due to conservation of the total magnetic moment. In this case the operator of the total spin commutes with the isotropic Kondo interaction and the latter disappears from the effective relaxation rate (see the review by Barnes [32]). Similar conclusions can be made, if the real parts of the kinetic coefficient $\Sigma_{s \sigma}$ and $\Sigma_{\sigma s}$ satisfy the conditions (23) (a field dominated bottleneck regime). In the opposite case of a strongly anisotropic Kondo interaction the results in the second order in the Kondo interaction do not show any sufficient narrowing of the ESR linewidth in the bottleneck regime [33].

The third order of the perturbation theory for the relaxation rates contain terms proportional to $\ln (W / T)$, what gives a divergent contribution at low temperatures $T \rightarrow 0$. It is especially important for the antiferromagnetic type of the Kondo interaction $\left(J_{\perp}, J_{\|}>0\right)$. The same terms appear in the scattering amplitudes of conduction electrons by magnetic impurities, what results in the Kondo effect in a resistivity. This phenomenon is also of a crucial importance for the ESR experiments. To reveal it one has to improve the perturbation theory for the spin relaxation rates.

\section{Renormalization of the spin relaxation rates}

To develop a satisfactory theory in the low-temperature regime one has to remove the logarithmic divergences by summing somehow the higher order terms in the perturbation expansion. Abrikosov [34] carried out a summation of the leading logarithmic terms for the resistivity applying the Feynman diagrams technique to the $s-d$ model. Later Anderson [35] proposed another method known as "Poor Man's Scaling" that allows one to extend the lowest order perturbation results to sum the leading order logarithmic terms on the basis of the Dyson equation. The main idea of the "Poor Man's Scaling" approach is to take into account the effect of the high-energy excitations on the low energy physics by a renormalization of coupling constants. Here we use this idea starting from the second order of the perturbation theory in the operator form suggested by Bogolubov and Tyablikov which was developed for the case of a degenerate ground energy level [36]. We apply it for the Kondo interaction in the form (12) in the absence of the external magnetic field

$$
\delta H_{K}=-P\left(H_{K}-P H_{K} P\right)\left(H_{0}-E_{0}\right)^{-1}\left(H_{K}-P H_{K} P\right) P .
$$

Here $P$ is a projecting operator of exited states onto the space of wave functions of the ground energy level. Substituting the explicit expression of the Kondo interaction, we obtain products of the $\mathrm{Yb}$ spin operators of the type $S_{i}^{\alpha} S_{j}^{\beta}$. In the case $i \neq j$ we obtain the RKKY interaction which was already taken into account by $H_{\mathrm{ex}}(10)$. In the case ( $i=j, S=1 / 2$ ) these products can be reduced to linear forms of spin operators:

$$
\begin{gathered}
S_{j}^{+} S_{j}^{-}=\frac{1}{2}+S_{j}^{z}, \quad S_{j}^{-} S_{j}^{+}=\frac{1}{2}-S_{j}^{z}, \quad\left(S_{j}^{z}\right)^{2}=\frac{1}{4}, \\
\left(S_{j}^{+}\right)^{2}=\left(S_{j}^{-}\right)^{2}=0, \quad S_{j}^{-} S_{j}^{z}=-S_{j}^{z} S_{j}^{-}=\frac{1}{2} S_{j}^{-}, \\
S_{j}^{+} S_{j}^{z}=-S_{j}^{+} S_{j}^{z}=-\frac{1}{2} S_{j}^{-} .
\end{gathered}
$$

The terms, which do not contain the $\mathrm{Yb}$ spin operators, can be related to renormalized conduction electrons energy. The rest operator of the second order contribution for the $j$-site is the following:

$$
\begin{aligned}
& \delta H_{K j}=\frac{1}{2} \sum_{\mathbf{k}_{1} \mathbf{k}_{2}} \sum_{\mathbf{k}_{3} \mathbf{k}_{4}}\left\{J_{\perp}^{2} S_{j}^{z} F_{\mathbf{k}_{1} . \mathbf{k}_{4}}^{z}+J_{\perp} J_{\|}\left[S_{j}^{+} F_{\mathbf{k}_{1} . \mathbf{k}_{4}}^{-}+S_{j}^{-} F_{\mathbf{k}_{1} . . \mathbf{k}_{4}}^{+}\right]\right\} \frac{\mathrm{e}^{i\left(\mathbf{k}_{2}-\mathbf{k}_{1}+\mathbf{k}_{4}-\mathbf{k}_{3}\right) \mathbf{r}_{j}}}{\varepsilon_{\mathbf{k}_{3}}-\varepsilon_{\mathbf{k}_{4}}} ; \\
& F_{\mathbf{k}_{1} . . \mathbf{k}_{4}}^{z}=c_{\downarrow \mathbf{k}_{1}}^{+} c_{\uparrow \mathbf{k}_{2}} c_{\uparrow \mathbf{k}_{3}}^{+} c_{\downarrow \mathbf{k}_{4}}-c_{\uparrow \mathbf{k}_{1}}^{+} c_{\downarrow \mathbf{k}_{2}} c_{\downarrow \mathbf{k}_{3}}^{+} c_{\uparrow \mathbf{k}_{4}}, \\
& 2 F_{\mathbf{k}_{1} \cdot . \mathbf{k}_{4}}^{-}=\left(c_{\uparrow \mathbf{k}_{1}}^{+} c_{\uparrow \mathbf{k}_{2}}-c_{\downarrow_{\mathbf{k}_{1}}}^{+} c_{\downarrow \mathbf{k}_{2}}\right) c_{\downarrow_{\mathbf{k}_{3}}}^{+} c_{\uparrow \mathbf{k}_{4}}-c_{\downarrow \mathbf{k}_{1}}^{+} c_{\uparrow} \mathbf{k}_{2}\left(c_{\uparrow \mathbf{k}_{3}}^{+} c_{\uparrow \mathbf{k}_{4}}-c_{\downarrow_{\mathbf{k}_{1}}}^{+} c_{\downarrow \mathbf{k}_{2}}\right), \\
& 2 F_{\mathbf{k}_{1} . \mathbf{k}_{4}}^{+}=c_{\uparrow \mathbf{k}_{1}}^{+} c_{\downarrow \mathbf{k}_{2}}\left(c_{\uparrow \mathbf{k}_{3}}^{+} c_{\uparrow} \mathbf{k}_{4}-c_{\downarrow \mathbf{k}_{1}}^{+} c_{\downarrow \mathbf{k}_{2}}\right)-\left(c_{\uparrow \mathbf{k}_{1}}^{+} c_{\uparrow \mathbf{k}_{2}}-c_{\downarrow \mathbf{k}_{1}}^{+} c_{\downarrow \mathbf{k}_{2}}\right) c_{\uparrow \mathbf{k}_{3}}^{+} c_{\downarrow \mathbf{k}_{4}} .
\end{aligned}
$$

We reduce the fourfold products of the creation and annihilation operators of conduction electrons to quadratic forms using the following type of approximations for terms diagonal in spin projections: 


$$
\begin{gathered}
c_{\downarrow \mathbf{k}_{1}}^{+} c_{\uparrow \mathbf{k}_{2}} c_{\uparrow \mathbf{k}_{3}}^{+} c_{\downarrow \mathbf{k}_{4}} \approx \delta_{\mathbf{k}_{1} \mathbf{k}_{4}}\left\langle c_{\downarrow_{\mathbf{k}_{1}}}^{+} c_{\downarrow \mathbf{k}_{4}}\right\rangle c_{\uparrow_{\mathbf{k}_{2}}} c_{\uparrow \mathbf{k}_{3}}^{+}+ \\
+\delta_{\mathbf{k}_{2} \mathbf{k}_{3}}\left\langle c_{\uparrow \mathbf{k}_{2}} c_{\uparrow \mathbf{k}_{3}}^{+}\right\rangle c_{\downarrow \mathbf{k}_{1}}^{+} c_{\downarrow \mathbf{k}_{4}}= \\
=-\delta_{\mathbf{k}_{1} \mathbf{k}_{4}} f_{\mathbf{k}_{1}} c_{\uparrow \mathbf{k}_{3}}^{+} c_{\uparrow_{\mathbf{k}_{2}}}+\delta_{\mathbf{k}_{2} \mathbf{k}_{3}}\left(1-f_{\mathbf{k}_{3}}\right) c_{\downarrow \mathbf{k}_{1}}^{+} c_{\downarrow \mathbf{k}_{4}} .
\end{gathered}
$$

Here $f_{\mathbf{k}}$ is the Fermi function. For nondiagonal terms we make similar approximations

$$
\begin{gathered}
\left(c_{\uparrow \mathbf{k}_{1}}^{+} c_{\uparrow \mathbf{k}_{2}}-c_{\downarrow_{\mathbf{k}_{1}}}^{+} c_{\downarrow \mathbf{k}_{2}}\right) c_{\downarrow \mathbf{k}_{3}}^{+} c_{\uparrow \mathbf{k}_{4}} \approx \\
\approx\left[\delta_{\mathbf{k}_{1} \mathbf{k}_{4}}\left\langle c_{\uparrow_{\mathbf{k}_{1}}}^{+} c_{\uparrow}\right) c_{\mathbf{k}_{1}}\right\rangle \uparrow_{\mathbf{k}_{2}} c_{\downarrow_{\mathbf{k}_{3}}}^{+}- \\
\left.-\delta_{\mathbf{k}_{2} \mathbf{k}_{3}}\left\langle c_{\downarrow \mathbf{k}_{2}} c_{\downarrow \mathbf{k}_{3}}^{+}\right\rangle c_{\downarrow \mathbf{k}_{1}}^{+} c_{\uparrow \mathbf{k}_{4}}\right]= \\
=-\delta_{\mathbf{k}_{1} \mathbf{k}_{4}} f_{\mathbf{k}_{1}} c_{\downarrow \mathbf{k}_{3}}^{+} c_{\uparrow_{\mathbf{k}_{2}}}-\delta_{\mathbf{k}_{2} \mathbf{k}_{3}}\left(1-f_{\mathbf{k}_{3}}\right) c_{\downarrow_{\mathbf{k}_{1}}} c_{\uparrow \mathbf{k}_{4}} .
\end{gathered}
$$

The result of these approximations is the following:

$$
\begin{aligned}
& H_{K j}=\frac{1}{2} \sum_{\mathbf{k}_{1} \mathbf{k}_{2}}\left[\delta J_{\perp}\left(S_{j}^{+} c_{\mathbf{k}_{1} \downarrow}^{+} c_{\mathbf{k}_{2} \uparrow}+S_{j}^{-} c_{\mathbf{k}_{1} \uparrow}^{+} c_{\mathbf{k}_{2} \downarrow}\right)+\delta J_{\|} S_{j}^{z}\left(c_{\mathbf{k}_{1} \uparrow}^{+} c_{\mathbf{k}_{2} \uparrow}-c_{\mathbf{k}_{1} \downarrow}^{+} c_{\mathbf{k}_{2} \downarrow}\right)\right] \mathrm{e}^{i\left(\mathbf{k}_{2}-\mathbf{k}_{1}\right) \mathbf{r}_{j}} \\
& \delta J_{\perp}=\frac{1}{2} \sum_{\mathbf{k}_{3}} J_{\perp} J_{\|}\left[\frac{1-2 f_{\mathbf{k}_{3}}}{\varepsilon_{\mathbf{k}_{3}}-\varepsilon_{\mathbf{k}_{2}}}-\frac{f_{\mathbf{k}_{3}}}{\varepsilon_{\mathbf{k}_{3}}-\varepsilon_{\mathbf{k}_{1}}}+\frac{f_{\mathbf{k}_{3}}}{\varepsilon_{\mathbf{k}_{3}}-\varepsilon_{\mathbf{k}_{2}}}\right]=\frac{J_{\|}}{J_{\perp}} \delta J_{\|} .
\end{aligned}
$$

The operator structure of this result is exactly the same as the starting Kondo interaction (12). The divergent contribution to $\delta J$ at $T \rightarrow 0$ comes from the first term in the second line in (29). Taking into account the relation $1-2 f(\varepsilon)=\tanh (\varepsilon / 2 T)$, we make the following approximation for this function: $\tanh x \approx x \quad(x<1)$, and $\tanh x \approx 1$ $(x>1)$. An approximate result for the sum in the second line in (29) is the following:

$$
\begin{gathered}
\frac{1}{N} \sum_{\mathbf{k}_{3}} \frac{1-2 f_{\mathbf{k}_{3}}}{\varepsilon_{\mathbf{k}_{3}}-\varepsilon_{\mathbf{k}_{2}}}=\int_{-W}^{W} d \varepsilon \rho(\varepsilon) \frac{\tanh (\varepsilon / 2 T)}{\varepsilon-\varepsilon_{2}}= \\
=\int_{0}^{W} d \varepsilon \rho(\varepsilon) \frac{2 \varepsilon \tanh (\varepsilon / 2 T)}{\varepsilon^{2}-\varepsilon_{2}^{2}} \approx \\
\approx \frac{\rho}{T} \int_{0}^{2 T} d \varepsilon \frac{\varepsilon^{2}}{\varepsilon^{2}-\varepsilon_{2}^{2}}+\rho \int_{2 T}^{W} d \varepsilon \frac{2 \varepsilon}{\varepsilon^{2}-\varepsilon_{2}^{2}} \approx 2 \rho \ln \left(\frac{W}{2 T}\right) .
\end{gathered}
$$

Here $\rho$ is the density of states at the Fermi surface; the populated level is expected to be $\varepsilon_{2}<2 T$. The main contribution comes from the second integral in the range $2 T<\varepsilon<W$. The other terms can be neglected in a comparison with the divergent one. As the result we have the following equations for the contribution of the second order:

$$
\delta J_{\|}=\rho J_{\perp}^{2} \ln \left(\frac{W}{2 T}\right), \quad \delta J_{\perp}=\frac{J_{\|}}{J_{\perp}} \delta J_{\|} .
$$

As the next step we divide the conduction electrons band into the low and high energy states

$$
0<\left|\varepsilon_{\mathbf{k}}\right|<\bar{W}, \quad \bar{W}<\left|\varepsilon_{\mathbf{k}}\right|<W,
$$

where $W, \bar{W}$ are the initial and the running half of the bandwidth, respectively. Using the Anderson's idea, we want to incorporate the contribution of the high-energy levels into renormalized values of $\bar{J}_{\perp}, \bar{J}_{\|}$. According to (31) it gives the following relations:

$$
\begin{gathered}
\delta \bar{J}_{\|}=\bar{J}_{\perp}^{2}\left[\ln \left(\frac{W}{2 T}\right)-\ln \left(\frac{\bar{W}}{2 T}\right)\right]=\rho \bar{J}_{\perp}^{2} \ln \left(\frac{W}{\bar{W}}\right), \\
\bar{J}_{\|} \delta \bar{J}_{\|}=\bar{J}_{\perp} \delta \bar{J}_{\perp} .
\end{gathered}
$$

These two equations represent the scaling law. From calculations (30) it follows that equations (33) are valid only for the range $(2 T<\bar{W}<W)$. The projection of the original Kondo interaction (12) on the low-energy states yields a new Hamiltonian of the same operator structure, but with renormalized coefficients. For a solution of the equations (33) we start from the second one. A simple integration gives

$$
\bar{J}_{\perp}^{2}-\bar{J}_{\|}^{2}=\text { const. }
$$

This relation was obtained first by Anderson for $T=0$ [35]. Since this equation does not depend on the value of the bandwidth, we choose the original values: const $=J_{\perp}^{2}-J_{\|}^{2}$. As the next step we take a small difference $W-\bar{W}=\delta \bar{W}$. It is convenient to introduce dimensionless values $\bar{U}_{\perp}=\rho \bar{J}_{\perp}$ and $\bar{U}_{\|}=\rho \bar{J}_{\|}$. Then according to (33) and (34) we have

$$
\begin{gathered}
\delta \bar{U}_{\|}=\bar{U}_{\perp}^{2} \ln \left(\frac{\bar{W}+\delta \bar{W}}{\bar{W}}\right) \approx \bar{U}_{\perp}^{2} \frac{\delta \bar{W}}{\bar{W}}, \\
\bar{U}_{\perp}^{2}-\bar{U}_{\|}^{2}=U_{0}^{2}=\rho\left(J_{\perp}^{2}-J_{\|}^{2}\right) .
\end{gathered}
$$

After substitution of the second equation into the first one we obtain a closed differential equation, which can be easily integrated:

$$
\begin{gathered}
\int_{U_{\|}}^{\tilde{U}_{\|}} \frac{\delta \bar{U}_{\|}}{\bar{U}_{\|}^{2}+U_{0}^{2}}=\int_{2 T}^{W} \frac{\delta \bar{W}}{\bar{W}} ; \\
\frac{1}{U_{0}}\left[\operatorname{arccot}\left(\frac{U_{\|}}{U_{0}}\right)-\operatorname{arccot}\left(\frac{\tilde{U}_{\|}}{U_{0}}\right)\right]=\ln \frac{W}{2 T} .
\end{gathered}
$$


Here $\tilde{U}_{\|}$is the final result of the renormalization process. Let us introduce the values

$\varphi=\operatorname{arccot}\left(\frac{\tilde{U}_{\|}}{U_{0}}\right), \quad T_{\mathrm{GK}}=\frac{W}{2} \exp \left[-\frac{1}{U_{0}} \operatorname{arccot}\left(\frac{U_{\|}}{U_{0}}\right)\right]$.

Then the final result of the renormalization process can be presented in the following form:

$$
\tilde{U}_{\|}=U_{0} \cot \varphi, \quad \tilde{U}_{\perp}=U_{0} \frac{1}{\sin \varphi} .
$$

Since the Zeeman Hamiltonian of Yb ions (6) has the same operator structure as the Kondo interaction (9) with parameters, satisfying the relation $g_{\perp} / g_{\|}=J_{\perp} / J_{\|}$, we accept the same relation between the renormalized $g$ factors

$$
\tilde{g}_{\perp} / \tilde{g}_{\|}=\tilde{J}_{\perp} / \tilde{J}_{\|}=\tilde{U}_{\perp} / \tilde{U}_{\|}
$$

The characteristic temperature $T_{\mathrm{GK}}$ is obviously independent of the initial and the running values of parameters, representing a universal energy scale to govern all the low temperature physics involving the ground Kramers doublet. All physical quantities can be expressed in terms of the ratios $\left(T / T_{\mathrm{GK}}\right)$. For the isotropic case with $g_{\perp}=g_{\|}$the characteristic temperature $T_{\mathrm{GK}}$ reduces to the well-known formula for the Kondo temperature $T_{\mathrm{GK}}^{\text {iso }}=(W / 2) \exp (-1 / \rho J)$. This formula follows from the logarithmic approximation; a more accurate result for the isotropic case is $T_{K} \sim(J W)^{1 / 2} \exp (-1 / \rho J)$ [37]. It is appropriate to mention that the scaling equations (33) can be represented in an alternative form, if to introduce a variable $t=\ln (W / \bar{W})$. Then from (33) we have

$$
\begin{array}{ll}
\delta \bar{U}_{\|}=\bar{U}_{\perp}^{2} \frac{\delta \bar{W}}{W}=\bar{U}_{\perp}^{2} \delta t, & \delta \bar{U}_{\perp}=\bar{U}_{\perp} \bar{U}_{\|} \delta t ; \\
\frac{d \bar{U}_{\|}}{d t}=\bar{U}_{\perp}^{2}, & \frac{d \bar{U}_{\perp}}{d t}=\bar{U}_{\perp} \bar{U}_{\|} .
\end{array}
$$

In this form the scaling equations were given by Belov et al. [25].

The temperature-dependent parameters are logarithmically divergent at $T \rightarrow T_{\mathrm{GK}}: \tilde{U}_{\|}, \tilde{U}_{\perp} \sim 1 / \ln \left(T / T_{\mathrm{GK}}\right)$, the perturbative scaling approach begins to break down. Consequently, all results derived by this method are only valid for temperatures above $T_{\mathrm{GK}}$.

\section{The bottleneck regime in presence of the Kondo effect}

Using the expressions (38) for the temperaturedependent Kondo couplings we find the renormalized kinetic coefficients

$$
\begin{array}{ll}
\tilde{\Gamma}_{s s}=\pi U_{0}^{2} T\left(\cot ^{2} \varphi+\frac{3}{4}\right), & \tilde{\Gamma}_{\sigma \sigma}=\frac{g_{\perp}}{2 g_{\sigma} \rho(T+\theta)} \tilde{\Gamma}_{s s}, \\
\tilde{\Gamma}_{s \sigma}=\frac{g_{\perp}}{2 g_{\sigma} \rho(T+\theta)} \tilde{\Gamma}_{\sigma s}, \quad \tilde{\Gamma}_{\sigma s}=\frac{\pi}{4} U_{0}^{2} T \frac{1}{\sin ^{2}(\varphi / 2)} .
\end{array}
$$

One can see that all kinetic coefficients logarithmically diverge at $T \rightarrow T_{\mathrm{GK}}$ : to the leading order in logarithmic terms they are of the form

$$
\begin{aligned}
& \tilde{\Gamma}_{s s}=\tilde{\Gamma}_{\sigma s}=\pi \frac{T}{\ln ^{2}\left(T / T_{\mathrm{GK}}\right)}, \\
& \tilde{\Gamma}_{\sigma \sigma}=\tilde{\Gamma}_{s \sigma}=\tilde{\Gamma}_{s s} \frac{g_{\perp}}{2 g_{\sigma} \rho(T+\theta)} .
\end{aligned}
$$

One could think that such a behavior confirms the common belief that the ESR linewidth of Kondo ions is expected to be too large for its detection. However, the coupling between two spin systems makes the situation quite different. It is interesting to analyze a solution of the coupled equations (17) under the condition of the bottleneck regime (23), taking into account the renormalization of kinetic coefficients. The relaxation rate of the collective spin mode with a frequency close to the Kondo-ion resonance now is the following:

$$
\begin{gathered}
\Gamma_{\text {coll }}=\Gamma_{s L}+\Gamma_{s s}^{\mathrm{eff}}+\Gamma_{\sigma L}^{\mathrm{eff}} \\
\Gamma_{s s}^{\mathrm{eff}}=\tilde{\Gamma}_{s s}-\frac{\tilde{\Gamma}_{s \sigma} \tilde{\Gamma}_{\sigma s}}{\tilde{\Gamma}_{\sigma \sigma}}, \quad \Gamma_{\sigma L}^{\mathrm{eff}}=\Gamma_{\sigma L} \frac{\tilde{\Gamma}_{s \sigma} \tilde{\Gamma}_{\sigma s}}{\tilde{\Gamma}_{\sigma \sigma}^{2}} .
\end{gathered}
$$

It is important to consider the asymptotic behavior of an effective Korringa relaxation rate $\Gamma_{s s}^{\text {eff }}$ and an effective spin relaxation rate of conduction electrons to the lattice $\Gamma_{\sigma L}^{\text {eff }}$ at temperatures approaching to $T_{\mathrm{GK}}$. In this case their expressions are essentially simplified to be written explicitly as functions of temperature:

$$
\begin{aligned}
& \Gamma_{s S}^{\mathrm{eff}}=\frac{\pi}{8} U_{0}^{4} T \ln ^{2}\left(T / T_{\mathrm{GK}}\right), \\
& \Gamma_{\sigma L}^{\mathrm{eff}}=\rho(T+\theta) \frac{2 g_{\sigma}}{g_{\perp}} \Gamma_{\sigma L} .
\end{aligned}
$$

This result is remarkable: instead of being divergent according to (42), the effective Korringa relaxation rate is greatly reduced and goes to zero at $T \rightarrow T_{\mathrm{GK}}$. Although the Kondo interaction is strongly anisotropic, the Kondo effect leads to the common energy scale $T_{\mathrm{GK}}$ regulating the temperature dependence of different physical quantities. As a result of this common energy scale, all divergent terms in the kinetic coefficients (41) experience their complete mutual cancelation in the collective spin mode even in the case of a strongly anisotropic system. The effective relaxation rate of the conduction electrons to the lattice is also greatly reduced, becoming proportional to temperature similar to the usual Korringa relaxation rate. This reduction supports the conditions for the bottleneck regime (23).

It is reasonable to mention that the divergent physical values appear due to approximate calculations. Nevertheless these divergences indicate strongly increasing values and stimulate more accurate calculations. At the same time we can conclude that although the used scaling procedure did not remove divergent terms from the initial relaxation 
rates (22), it was able to remove them from the relaxation rates of the final solution for the collective modes having real physical sense.

Concerning the broadening of the ESR linewidth which is represented by the kinetic coefficient $\Gamma_{s L}$, one could expect an obvious contribution from the distribution of effective local magnetic fields due to spin-spin interactions of the $\mathrm{Yb}$ ions. In particular, the usual magnetic dipoledipole interactions yield approximately $\Delta H_{\text {loc }} \approx 700 \mathrm{Oe}$, while the observed ESR linewidth in $\mathrm{YbRh}_{2} \mathrm{Si}_{2}$ at the $X$ and $Q$ bands is $\Delta H_{\mathrm{ESR}} \approx 200 \mathrm{Oe}$ at $T=5 \mathrm{~K}$. Therefore, it is evident that some narrowing mechanism for these type of contributions should exist. At the same time, it is well known that the broadening of the ESR line by the distribution of local fields can be reduced in the bottleneck regime due to fast reorientation of the Kondo ion spins caused by the Korringa relaxation [32], the more as being greatly increased due to the Kondo effect. Another reason for the relaxation rate $\Gamma_{s L}$ can be related to the spin-phonon interactions. At temperatures above a few $\mathrm{K}$ the main contribution comes from the two-phonon processes. The Orbach process via the excited energy level at a given crystal field splitting $\Delta$ yields the temperature dependence

$$
\Gamma_{s L}^{\mathrm{Orb}} \sim[\exp (\Delta / T)-1]^{-1}
$$

This type of contribution does not need any scaling procedure.

In the case of the ESR resonance frequency of the collective mode, the situation is somewhat different. For a single Kondo ion it is well known that besides the usual Knight shift of the ESR resonance frequency, the Kondo effect results in a divergent logarithmic term. The same happens with the resonance frequency of the conduction electrons. We have found that all divergent parts of the ESR resonance frequency cancel each other in the collective mode similar to the relaxation rate described above. However, the RKKY interaction provides an additional local field at the $\mathrm{Yb}$ ion and the Weiss constant $\theta$ in the spin susceptibility. In the molecular field approximation involving both the Kondo and RKKY interactions, $\theta$ becomes also a subject of the Kondo renormalization. As a result, the ESR resonance frequency contains a divergent logarithmic term even for the collective spin mode. For the corresponding effective $g$ factor $g_{\perp}^{\text {eff }}$ the following relation was obtained for the magnetic field perpendicular to the symmetry axis [23]:

$$
\frac{g_{\perp}}{g_{\perp}^{\text {eff }}}=1+\frac{\theta}{T}\left\{1+U_{\|}+U_{0}\left[\frac{1}{2} \operatorname{arccot}\left(\frac{U_{\|}}{U_{0}}\right)-\cot \varphi\right]\right\} \text {. }
$$

Next we consider the variation of the ESR parameters with the orientation of the microwave magnetic field having the angle $\eta$ relative to the plane perpendicular to the $c$ axis [25]. The static magnetic field is still perpendicular to the $c$ axis and microwave field. It was found that the $g$ factor $g_{\perp}$ and the relaxation rates (41) do not depend on the orientation of the microwave magnetic field. It is obvious that the relaxation rate of the collective spin mode given by (43) and the resonant frequency are also independent of the angle $\eta$.

Concerning the ESR intensity, the situation is quite different. It is known that the intensity is determined by the $g$ factor component along the direction of the microwave magnetic field: $I \sim g_{\|}^{2} \sin ^{2} \eta+g_{\perp}^{2} \cos ^{2} \eta$. Taking into account the renormalization of the $g$-factor components in accordance with (39), it was obtained for renormalized ESR intensity

$$
\tilde{I}(\eta, T)=\tilde{I}(0, T)\left[1-\sin ^{2} \varphi \sin ^{2} \eta\right]
$$

where $\varphi$ is defined by (37). The ratio $\tilde{I}(0, T)=\tilde{I}(\pi / 2, T)$ is reduced to $1 / \cos ^{2} \varphi$ instead of the values $g_{\perp}^{2} / g_{\|}^{2} \approx 14$ and $g_{\perp}^{2} / g_{\|}^{2} \approx 400$ in the cases of $\mathrm{YbIr}_{2} \mathrm{Si}_{2}$ and $\mathrm{YbRh}_{2} \mathrm{Si}_{2}$, respectively.

It is reasonable to expect, that since the all divergent terms at $T \rightarrow T_{\mathrm{GK}}$ were mutually canceled in kinetic coefficients of the collective modes, the obtained result can be valid even at temperatures close to the $T_{\mathrm{GK}}$.

\section{Experimental ESR results}

As a matter of fact, the ESR measurements with very interesting results were performed first at every step and then have stimulated the theoretical investigations described in the previous sections. At first, we use the divergent logarithmic term in the $g$ factor (46) to reveal the characteristic temperature $T_{\mathrm{GK}}$. As the starting value for the temperature dependence of the $g$ factor was taken temperature-independent experimental result at high temperatures $g_{\perp}=3.65$, the density of states can be related to the band width of the conduction electrons as $\rho=1 / W$. The result of the fitting is given in Fig. 4(a) with $U_{0}=0.18$, $\theta \approx 0.2 \mathrm{~K}$ and $T_{\mathrm{GK}}=0.36 \mathrm{~K}$; the latter is by two orders of magnitude smaller than the Kondo temperature $T_{K}$ derived thermodynamically [38] and by transport measurements [39]. The revealed value $T_{\mathrm{GK}}$ was used to fit the temperature and frequency dependences of the ESR linewidth with help of equations (43) in order to see whether the theory is selfconsistent:

$$
\Gamma_{\text {theor }}=\Gamma_{\text {coll }}+\Gamma_{s L}^{\mathrm{Orb}}+\text { const. }
$$

Here const represents the local field distribution which is greatly reduced as discussed above. The results are given in Fig. 4(b). The fitting of the temperature dependence of the ESR linewidth gave $U_{0}$ about the same and $\Delta=198 \mathrm{~K}$. The latter coincides with the first excited energy level of the $\mathrm{Yb}$ ion [20], confirming that the Orbach processes dominate in the spin-phonon relaxation. Having the value $U_{0}=0.18$, we can now estimate the Korringa relaxation rate without the bottleneck regime. According to equation (41) this value yields $\Gamma_{s s} \approx 50 \mathrm{GHz}$ at $5 \mathrm{~K}$. Such 


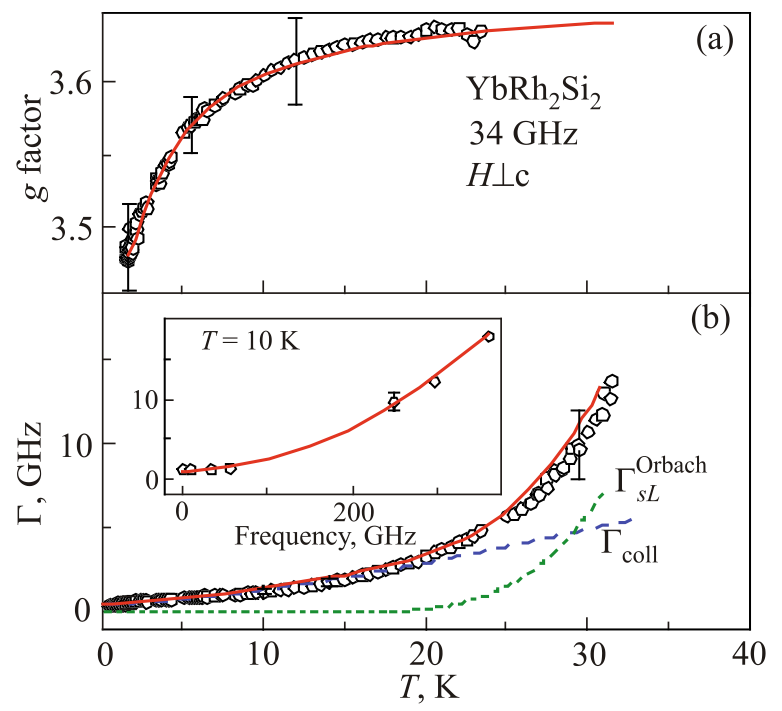

Fig. 4. (Color online) Temperature dependence of $Q$ band (a) $g$ factor and (b) ESR relaxation rate $\Gamma$ of $\mathrm{YbRh}_{2} \mathrm{Si}_{2}$ (open circles) from [23]. Solid lines denote data fitting: $g_{\perp}^{\text {eff }}$ (Eq. (46)) and $\Gamma_{\text {theor }}$ (Eq. (48)) with two contributions $\Gamma_{\text {coll }}$ and $\Gamma_{S L}^{\text {Orbach }}$ as indicated. Inset: frequency dependence of $\Gamma_{\text {theor }}$ (solid line) fitted to $\Gamma(T=10 \mathrm{~K})$.

a linewidth would leave no chance to observe the ESR signal, neither at $X$ or $Q$ bands nor at higher frequencies without formation of the collective spin mode.

Next we compare the developed theory with the experimental data for the case of an arbitrary orientation of the microwave field with respect to basal plane [25]. The static field was always perpendicular to the $c$ axis ( $\eta$ is varying). The resonant frequency and the relaxation rate were predicted to be independent of the angle what is in full agreement with experimental data of $\mathrm{YbRh}_{2} \mathrm{Si}_{2}$ and $\mathrm{YbIr}_{2} \mathrm{Si}_{2}$ (see Fig. 5). The angular dependence of the ESR intensity is fitted with the theoretical expression (47). The results of

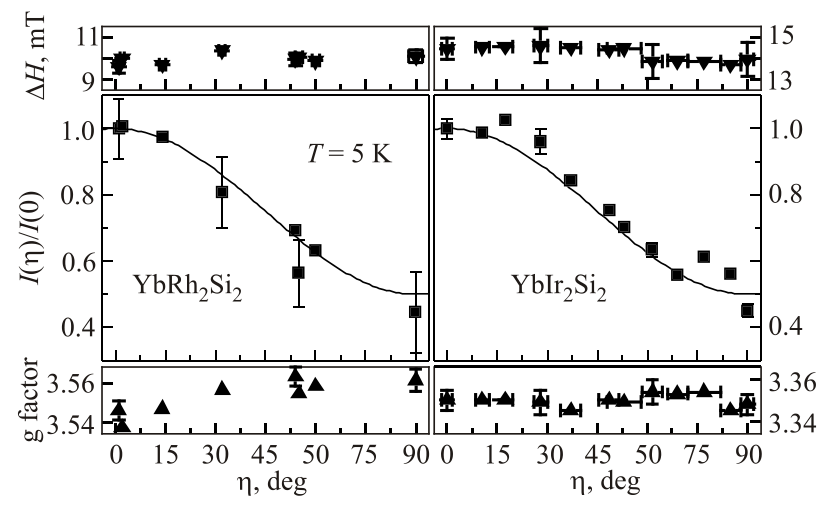

Fig. 5. Variation of the ESR intensity of $\mathrm{YbRh}_{2} \mathrm{Si}_{2}$ and $\mathrm{YbIr}_{2} \mathrm{Si}_{2}$ by changing the angle $\eta$ only and keeping the static field always perpendicular to the $c$ axis (from [25]). The solid lines denote data fitting according to Eq. (47) with the ratio $\tilde{I}(\eta) / \tilde{I}(0)$. The upper and lower graphs show the corresponding linewidth $\Delta H$ and the $g$ factor $g_{\perp}$, respectively. Details on the intensity determination are given in [7]. the fitting are given in Fig. 5: the intensity of both compounds can be described with the same value $\sin ^{2} \varphi \approx 0.5$. The parameters $U_{0}$ and $T_{\mathrm{GK}}$ could not be determined unambiguously because the experimental data on the angular dependence of the ESR intensity were obtained only for $T=5 \mathrm{~K}$.

\section{Concluding remarks}

The discovery of the electron spin resonance in the Kondo lattice with heavy fermions gave not only new opportunities to study these interesting materials but also stimulated new approaches in understanding of their complicated properties. It is evident that the conception based on the single Kondo ion effect does not work in the case of Kondo lattice. It is remarkable that the Kondo effect, being responsible for a suppression of the ESR signal on paramagnetic impurities in a metal at low temperatures, crucially supports it in the Kondo lattice due to the formation of the collective spin mode with a dramatic narrowing of the ESR linewidth. It happens even in the case of a strongly anisotropic Kondo interaction in contradiction with the case of a small concentration of the Kondo ions as impurities.

It is interesting to note that there is a deep similarity of this phenomenon with another one related to the ESR on paramagnetic impurities in superconductors. The first observations of the ESR have shown a sharp increase of the spin relaxation rate after transition into the superconducting state $[40,41]$. It was in a full agreement with results of the nuclear magnetic resonance (NMR) and the BardeenCooper-Schrieffer (BCS) theory. The well-known HebelSlichter peak appears due to increasing of the spin relaxation rate to conduction electrons in consequence of sharp increasing of their density of states at the energy gap near the Fermi level and a coherence factor of the Cooper paring. However, very soon an unexpected phenomenon was observed by ESR on erbium impurities in lanthanum: the ESR linewidth was sharply narrowed instead of an increase after the transition to the superconducting state [42]. In order to understand this phenomenon it was suggested that an increase of the coupling between the spin systems of impurities and superconducting electrons resulted in a transition into the bottleneck regime [43]. This idea was proved later by the direct analysis using the Feynman diagram technic [44]. In the case of the NMR the bottleneck regime cannot appear because of a great difference of electron and nuclear magnetic moments. As a matter of fact the phenomenon of the ESR narrowing in superconductors and in the Kondo lattice have a common nature: a formation of a collective modes of two spin subsystems due to enhanced coupling between them caused on the one hand by a transition to the superconducting state and by the Kondo effect on the other hand.

It is appropriate to mention some questions still waiting their elucidation. In particular, it is not clear how the characteristic temperature for the ground Kramers doublet $T_{\mathrm{GK}}$ 
relates to the thermodynamical Kondo temperature $T_{K}$. An analysis of the phase transition from the NFL state to the AFM state needs additional ESR experiments at very low temperatures and magnetic fields and theoretical efforts. There is no common understanding of the phase transition from the AFM state to the LFL state near the QCP.

\section{Acknowledgments}

I am very grateful to Ilya M. Lifshitz for his support at the beginning of my scientific carrier. Many thanks to J. Sichelschmidt, S.I. Belov, and A.S. Kutuzov for a help in preparation of the paper. This work is supported by the subsidy allocated to Kazan Federal University for the state assignment in the sphere of scientific activities.

1. P. Gegenwart, Y. Tokiwa, T. Westerkamp, F. Weickert, J. Custers, J. Ferstl, C. Krellner, C. Geibel, P. Kerschl, K.-H. Müller, and F. Steglich, New J. Phys. 8, 171 (2006).

2. J. Sichelschmidt, V.A. Ivanshin, J. Ferstl, C. Geibel, and F. Steglich, Phys. Rev. Lett. 91, 156401 (2003).

3. J. Sichelschmidt, J. Wykhoff, H.-A. Krug von Nidda, J. Ferstl, C. Geibel, and F. Steglich, J. Phys.: Condens. Matter 19, 016204 (2007).

4. J. Sichelschmidt, T. Kambe, I. Fazlishanov, D. Zakharov, H.-A. Krug von Nidda, J. Wykhoff, A. Skvortsova, S. Belov, A. Kutuzov, B.I. Kochelaev, V. Pashchenko, M. Lang, C. Krellner, C. Geibel, and F. Steglich, Phys. Status Solidi B 247, 747 (2010).

5. J. Wykhoff, J. Sichelschmidt, G. Lapertot, G. Knebel, J. Flouquet, I.I. Fazlishanov, H.-A. Krug von Nidda, C. Krellner, C. Geibel, and F. Steglich, Science Techn. Adv. Mat. 8, 389 (2007).

6. J. Sichelschmidt, J. Wykhoff, H.-A. Krug von Nidda, I.I. Fazlishanov, Z. Hossain, C. Krellner, C. Geibel, and F. Steglich, J. Phys.: Condens. Matter 19, 016211 (2007).

7. T. Gruner, J. Wykhoff, J. Sichelschmidt, C. Krellner, C. Geibel, and F. Steglich, J. Phys.: Condens. Matter 22, 135602 (2010).

8. U. Schaufuss, V. Kataev, A.A. Zvyagin, B. Buchner, J. Sichelschmidt, J. Wykhoff, C. Krellner, C. Geibel, and F. Steglich, Phys. Rev. Lett. 102, 076405 (2009).

9. A.A. Zvyagin and A.V. Makarova, J. Phys.: Condens. Matter 17, 1251 (2005).

10. A.A. Zvyagin, V. Kataev, and B. Buchner, Phys. Rev. B 80, 024412 (2009).

11. E. Abrahams and P. Wölfle, Phys. Rev. B 78, 104423 (2008).

12. P. Wölfle and E. Abrahams, Phys. Rev. B 80, 235112 (2009).

13. P. Schlottmann, Phys. Rev. B 79, 045104 (2009).

14. D.L. Huber, J. Phys.: Condens. Matter 21, 322203 (2009); ibid. 24, 226001 (2012); Mod. Phys. Lett. B 26, 1230021 (2012).

15. D.V. Vyalikh, S. Danzenbächer, Yu. Kucherenko, K. Kummer, C. Krellner, C. Geibel, M.G. Holder, T.K. Kim, C. Laubschat, M. Shi, L. Patthey, R. Follath, and S.L. Molodtsov, Phys. Rev. Lett. 105, 237601 (2010).
16. A.S. Kutuzov, A.M. Skvortsova, S.I. Belov, J. Sichelschmidt, J. Wykhoff, I. Eremin, C. Krellner, C. Geibel, and B.I. Kochelaev, J. Phys.: Condens. Matter 20, 455208 (2008).

17. A.S. Kutuzov and A.M. Skvortsova, Magn. Reson. Solids 11, 7 (2009).

18. A.S. Kutuzov and A.M. Skvortsova, J. Phys.: Conf. Ser. 324, 012039 (2011).

19. A. Abragam and B. Bleaney, Electron Paramagnetic Resonance of Transition Ions, Clarendon Press, Oxford (1970).

20. O. Stockert, M.M. Koza, J. Ferstl, A.P. Murani, C. Geibel, and F. Steglich, Physica B 378-380, 157 (2006).

21. Q. Si, S. Rabello, K. Ingersent, and L. Smith, Nature 413, 804 (2001).

22. P. Gegenwart, Y. Tokiwa, J. Custer, C. Geibel, and F. Steglich, J. Phys. Soc. Jpn. 75 (Suppl.), 155 (2006).

23. B.I. Kochelaev, S.I. Belov, A.M. Skvortsova, A.S. Kutuzov, J. Sichelschmidt, J. Wykhoff, C. Geibel, and F. Steglich, Eur. Phys. J. B 72, 485 (2009).

24. S.I. Belov, A.S. Kutuzov, and B.I. Kochelaev, J. Phys.: Conf. Ser. 324, 012017 (2011).

25. S.I. Belov, A.S. Kutuzov, B.I. Kochelaev, and J. Sichelschmidt, J. Phys.: Condens. Matter 24, 365601 (2012).

26. S.I. Belov and A.S. Kutuzov, Magn. Reson. Solids 14, 12103 (2012).

27. S.I. Belov and A.S. Kutuzov, Magn. Reson. Solids 16, 14103 (2014).

28. S.I. Belov and A.S. Kutuzov, Appl. Magn. Reson. 45, 1179 (2014).

29. S.I. Belov and A.S. Kutuzov, Magn. Reson. Solids 17, 15104 (2015).

30. S.I. Belov and A.S. Kutuzov, J. Low Temp. Phys. 185, 641 (2016).

31. K.N.R. Taylor, M.I. Darby, Physics of Rare Earth Solids, Chapman and Hall, London (1972).

32. S.E. Barnes, Adv. Phys. 30, 801 (1981).

33. B.I. Kochelaev and A.M. Safina, Phys. Solid State 46, 226 (2004).

34. A.A. Abrikosov, Physics 2, 5 (1965).

35. P.W. Anderson, J. Phys. C 3, 2436 (1970).

36. N.N. Bogolubov and S.V. Tyablikov, JETP 19, 251 (1949).

37. K. Wilson, Rev. Mod. Phys. 47, 773 (1975).

38. O. Trovarelli, C. Geibel, S. Mederle, C. Langhammer, F.M. Grosche, P. Gegenwart, M. Lang, G. Sparn, and F. Steglich, Phys. Rev. Lett. 85, 626 (2000).

39. U. Köhler, N. Oeschler, F. Steglich, S. Maquilon, and Z. Fisk, Phys. Rev. B 77, 104412 (2008).

40. T.S. Altshuler, I.A. Garifullin, E.G. Kharakhashyan, Phys. Solid State 14, 263 (1972).

41. C. Rettori, D. Davidov, P. Chaikin, and R. Orbach. Phys. Rev. Lett. 30, 437 (1973).

42. N.E. Alekseevsky, I.A. Garifullin, B.I. Kochelaev, and E.G. Kharakhashyan, JETP Lett. 18, 189 (1973) [Pisma Zh. Eksp. Teor. Fiz. 18, 323 (1973)].

43. B.I. Kochelaev, E.G. Kharakhashyan, I.A. Garifullin, and N.E. Alekseevsky, 18th AMPERE Congress, Nottingham (1974).

44. A.A. Kosov, B.I. Kochelaev, Phys. Solid State 74, 148 (1978). 